\section{Uncovering cryptic CAM}

J. Exp. Bot. https://doi.org/10.1093/jxb/ery431 (2018).

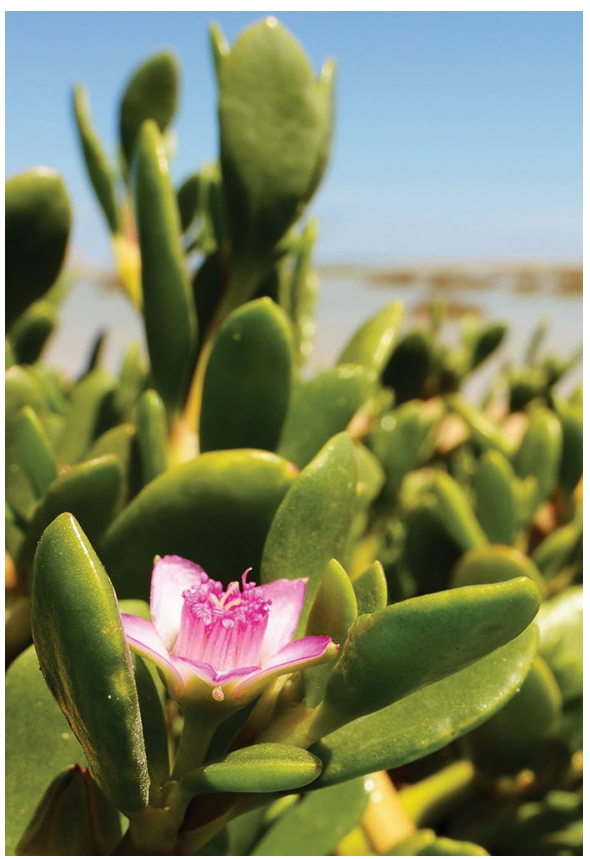

Credit: NICK UPTON / ALAMY STOCK PHOTO

Photosynthesis is not a simple process. There are two main forms, $\mathrm{C}_{3}$ and $\mathrm{C}_{4}$, and almost all plants use one or the other, or sometimes both; however, numerous subtle variations of each exist, making determining a plant's fundamental biochemistry fraught with difficulty. A case in point is the shoreline plant, sea purslane (Sesuvium portulacastrum). S. portulacastrum has smooth, fleshy leaves that are superficially similar to a succulent. However, it has taken extremely careful measurements by Klaus Winter and colleagues, from the Smithsonian Tropical Research Institute in Panama, to show that it can also employ the favourite form of photosynthesis for succulents, crassulacean acid metabolism (CAM), and only under highly stressful conditions.

CAM photosynthesis developed in land plants as an adaption to low water availability. Most plants open the stomata on their leaves during the day to allow for exchange of oxygen and carbon dioxide between actively photosynthesising tissues and the outside air, but close them during the night. With CAM, this pattern is reversed. Stomata close during the heat of the day to reduce water loss but open at night to take in carbon dioxide, which is stored as malic acid in cell vacuoles ready for use by photosynthesis when the sun returns. The consequent acidification of leaves during the night (as well as carbon dioxide uptake during those times) is thus characteristic of CAM.

S. portulacastrum is widely distributed throughout the world. It is native to Asia, Africa, Australia, North and South America, and is extensively naturalized elsewhere. In the Philippines, it is pickled and eaten as a chutney. Other Sesuvium species, as well as many plants within the wider Aizoaceae family, employ CAM, yet $S$. portulacastrum appeared to be resolutely a $\mathrm{C}_{3}$ plant. Winter et al. grew $S$. portulacastrum in outdoor plots in Panama and constantly measured the acidity of their leaves. The first months of experiments coincided with a period of high rainfall and no acidity was seen in the leaves, but in the last two months when the weather was dry, the leaves began to show reduced $\mathrm{pH}$. The researchers confirmed that carbon dioxide was being absorbed during the night, leading to the acidification, with pot-based experiments. These allowed for more controlled growing conditions and gas exchange measurements on individual plants. The transition to CAM only occurred under conditions of severe drought, serving as a short-term survival strategy.

The fact that $S$. portulacastrum can employ CAM in times of water stress is a further demonstration of the plasticity of plant metabolisms in adapting to the wildly changing conditions that the sedentary lifestyles of plants forces them to endure.

\section{Chris Surridge}

Published online: 9 January 2019 https://doi.org/10.1038/s41477-018-0351-2 